\section{A Atuação da Fisioterapia no Tratamento de Sequela de Queimaduras por Meio da Indução Percutânea de Colágeno: Estudo de Caso}

\section{Resumo}

O presente estudo se propôs a aplicar a técnica de Indução Percutânea de Colágeno (IPC) em uma cicatriz causada por queimadura de segundo grau profundo na região anterior do braço esquerdo. Foram realizadas três sessões de IPC, com intervalo de 30 dias entre cada uma das sessões. As sessões foram realizadas em uma Clínica de Fisioterapia localizada no Hospital Santa Cruz, em São Paulo, mesmo local onde foi realizada a anamnese, aplicação da escala de Vancouver, aplicação da Escala Visual Numérica e Registro Fotográfico. Os resultados obtidos pela escala de Vancouver revelam melhora, sendo que o aspecto avaliado que mais apresentou meIhora foi a flexibilidade da pele, seguido da vascularização e altura da cicatriz. Não foi possível observar melhora na pigmentação da cicatriz através da escala de Vancouver. O score total passou de 7 para 4 após a última sessão, diminuindo em 3 pontos no total (cerca de 43\%). Para avaliação da pigmentação da pele foi aplicado questionário elaborado pela pesquisadora. O questionário foi respondido por oito avaliadores cegos e apresentou melhora geral entre todos os participantes de aproximadamente 36\%. A Escala Visual Numérica apresentou aumento na sensibilidade da pele.

Palavras-Chave: Cicatriz; Queimadura; Indução Percutânea de Colágeno; Microagulhamento; Tratamento.
Prof ${ }^{a}$ Me. Pamela

Barbosa Arantes

Mestre em Ciência e

Tecnologia em Saúde, Cruzeiro do Sul Virtual.

E-mail: pamela.arantes @cruzeirodosul.edu.br

Prof. ${ }^{a}$ Dr. ${ }^{a}$ Kátia Cristina Ugolini Mugnol

Pós-Doutora em Biossistemas pela Universidade Federal do ABC, docente na Universidade Mogi das Cruzes.

E-mail: katiac

@umc.br.

Prof. ${ }^{\text {a Dr. }}$. Vilma da Natividade da Silva Santos

Doutora em Ciências da Saúde pela Faculdade de Medicina do ABC.

E-mail: vilma.natividade @gmail.com. 


\section{Abstract:}

The present study proposed to apply the Percutaneous Colagen Induct (PCI) in a scars caused by second degree burns in left arm. Were performed 3 sessions of PCI, with an interval of 30 days between them. The interventions were performed at Physical Therapy Clinic, located at Santa Cruz Hospital, in São Paulo, the same place where was performed the anamnesis, the application of Vancouver Scale and Visual Numerical Scale and also the photographic recording. The results obtained by using the Vancouver Scale show improvement, and the aspect it was most evident was the flexibility of the skin, followed by the vascularization and the height of the scar. The total score among participants went from 7 to 4 after the last session, decreasing by 3 points in total (about 43\%). As it was not possible to observe improvement in scar pigmentation through the Vancouver Scale, a blind assessment was performed by applying a questionnaire prepared by the researcher to 8 evaluators with proven knowledge in the research area. This assessment determined that $P C I$ resulted in improvement of this parameter in treated lesions, with approximately $36 \%$ skin whitening. To evaluate skin sensitivity we used the Visual Numerical Scale, which showed promising results also represented by increased local sensitivity.

Keywords: Scar; Burn; Percutaneous Collagen Induction; Microneedling; Treatment.

\section{Introdução}

As queimaduras ainda são responsáveis por grande parte dos ferimentos e óbitos decorrentes de causas externas no Brasil, bem como por grande número de afastamentos de funcionários do trabalho e de sequelas funcionais e estéticas (METSAVAHT, 2017). São lesões cutâneas causadas pela ação direta ou indireta do calor e as principais causas são a chama direta, o contato com água fervente ou líquidos quentes (chamado de escaldamento), o contato com superfícies aquecidas, acidentes com corrente elétrica e agentes químicos (VALE, 2015). Grande porcentagem de acidentes que resultam em queimaduras ocorre em ambiente domiciliar (RIBEIRO, 2013).

A definição de queimadura está diretamente relacionada às agressões pelo calor, o que provoca lesão da pele com coagulação direta dos tecidos e perda da barreira cutânea. Podemos, porém, ampliar essa definição e classificar a queimadura como a perda dos tecidos cutâneos tanto pela ação de fatores térmicos quanto químicos, elétricos ou radiação. Portanto, pode-se definir que a queimadura pode ocorrer pela ação de calor, frio, abrasão, produtos ácidos, álcalis e corrosivos, passagem da corrente elétrica ou radiação nuclear, levando a diferentes fisiopatologias e manifestações clínicas (STEFANELLI, 2015).

O tratamento das queimaduras é bem estabelecido nos hospitais gerais e especializados e se estende para além do cuidado imediato, já que a maioria das queimaduras deixa sequelas que variam de pequenas cicatrizes a limitações importantes de movimento. O tratamento dessas sequelas é bastante complexo e justifica que se busquem alternativas que possam não somente melhorar o aspecto das cicatrizes, mas também, principalmente, melhorar a qualidade de vida do paciente.

A Indução Percutânea de Colágeno (IPC) pode ser uma alternativa interessante no tratamento das sequelas de queimaduras, já que é um procedimento pouco invasivo e de relativo baixo custo. Há, entretanto, escassez na literatura sobre aplicação dessa técnica nesse tipo de lesão, sendo dado mais enfoque em seu uso para condições estéticas, como no tratamento de cicatrizes causadas por quadros de acne, rejuvenescimento, alterações de pigmentação da pele e, ainda, pouquíssimos trabalhos em estrias.

Acreditando-se no potencial dessa técnica para a melhora tanto da aparência da sequela de queimadura quanto na melhoria de fatores que podem ampliar a autoestima e o bem-estar desses pacientes, buscou-se, por meio do presente estudo, determinar se a aplicação da IPC em uma paciente com cicatrizes de queimadura há 1 ano e dois meses levaria à melhora no bem-estar e à satisfação dessa paciente, por meio da melhora 
da sensibilidade, pigmentação, flexibilidade, vascularização e altura da cicatriz.

\section{Queimadura}

Para a Sociedade Brasileira de Queimadura, atualmente, a maior ocorrência de acidentes por queimadura no Brasil acontece por contato em superfícies superaquecidas, como ferro de passar roupa, tampa de panela, forno, churrasqueira, escapamento de motocicletas, entre outros. Isso supera índices anteriores, que apontavam para líquidos superaquecidos (SILVA, 2016).

A queimadura de espessura superficial ou de $1^{\circ}$ grau é a que atinge somente a camada epidérmica; é clinicamente caracterizada por eritema, edema e dor. Na evolução há prurido e descamação. A queimadura de espessura parcial superficial ou de $2^{\circ}$ grau superficial é a que atinge toda a epiderme e parte da derme, em especial a derme papilar, conservando parcialmente os apêndices epidérmicos. Clinicamente, é caracterizada por vesícula, eritema, dor acentuada, edema e apresenta a base das bolhas avermelhada. A pele sofre processo de restauração completa dentro de 7 a 10 dias. Já a queimadura de espessura parcial profunda ou de $2^{\circ}$ grau profundo é a que atinge toda a epiderme e a derme mais profundamente, até a derme reticular, mantendo ainda parcialmente os apêndices epidérmicos. Contudo, clinicamente, não apresenta dor e a base das bolhas é esbranquiçada. Pode ocorrer restauração, mas há alterações de textura e pigmentação (FERREIRA, 2007).

A queimadura de espessura total, ou de $3^{\circ}$ grau, ocorre quando foram destruídas todas as camadas da pele, podendo atingir tecidos profundos do corpo humano. Clinicamente, é caracterizada por lesão dura e seca, de coloração esbranquiçada ou escura, ausência de vesículas e de dor. O tratamento é cirúrgico para limpeza e a cobertura das lesões pela ausência total de apêndices epidérmicos (FERREIRA, 2007).

As lesões pós-queimadura são classificadas em: (a) sequelas funcionais, aquelas que limitam qualquer função de um segmento após uma queimadura, e (b) as sequelas estéticas ou não funcionais, que são as que não comprometem a funcionalidade, mas que podem interferir nos aspectos sociais decorrentes da nova aparência (VANA, 2013).

Para melhor compreender os efeitos de uma queimadura, é necessária breve revisão sobre a pele, suas estruturas e funções, características da pele queimada, apresentada a seguir.

\section{Pele}

A pele deriva dos folhetos embrionários ectoderme e mesoderme. As estruturas epiteliais de origem ectodérmica são as unidades pilossebáceas, glândulas apócrinas, glândulas écrinas e unhas. Os melanócitos e nervos derivam de um segmento específico da ectoderme, a neuroectoderme. A mesoderme origina os fibroblastos, vasos sanguíneos, músculos e adipócitos (PETRI, 2009).

A pele é constituída, basicamente, por três camadas interdependentes: a epiderme, mais externa; a derme, intermediária; e a hipoderme, mais profunda (figuras 1 e 2). A transição entre a epiderme e a derme é denominada junção dermoepidérmica ou zona da membrana basal (AZULAY, 2017).

A pele do ser humano, que corresponde a $15 \%$ de seu peso corporal, é um órgão que reveste e delimita o organismo, protegendo-o e interagindo com o meio exterior. Sua resistência e flexibilidade determinam a sua plasticidade. Essencialmente dinâmica, a pele apresenta alterações constantes, sendo dotada de grande capacidade renovadora e de reparação, e de certo grau de impermeabilidade. Sua mais importante e vital função é a conservação da homeostasia (termorregulação, controle hemodinâmico e produção e excreção de metabólitos). Desempenha, ainda, função sensorial, por intermédio dos elementos do sistema nervoso situado na derme, e função de defesa contra agressões físicas, químicas e biológicas, para a qual se destacam, pela sua importância, a queratinizarão, o manto lipídico e o sistema imunológico (AZULAY, 2017). 


\section{Pele Queimada}

A pele humana pode tolerar, sem prejuízo, temperaturas de até $44^{\circ} \mathrm{C}$. Acima dessa temperatura, são produzidas diferentes lesões. O grau da lesão está relacionado à temperatura e ao tempo de exposição (BOLGIANI e SERRA, 2010).

As queimaduras são feridas traumáticas causadas, na maioria das vezes, por agentes térmicos, químicos, elétricos ou radioativos. Atuam nos tecidos de revestimento do corpo humano, determinando destruição parcial ou total da pele e de seus anexos, podendo acometer camadas mais profundas, como tecido celular subcutâneo, músculos, tendões e ossos (AZULAY, 2017).

Grande porcentagem de acidentes que resultam em queimaduras ocorre em ambiente domiciliar. Dentre as principais causas apontadas, estão escaldamento, fogo direto, contato com objetos quentes, eletricidade e exposição a agentes químicos (RIBEIRO, 2013).

A classificação quanto à profundidade da queimadura é comumente dada em graus (primeiro, segundo e terceiro graus). Determinar - grau de queimadura significa determinar a profundidade do trauma na pele (GUIRRO e GUIRRO, 2007) (Figura 1).

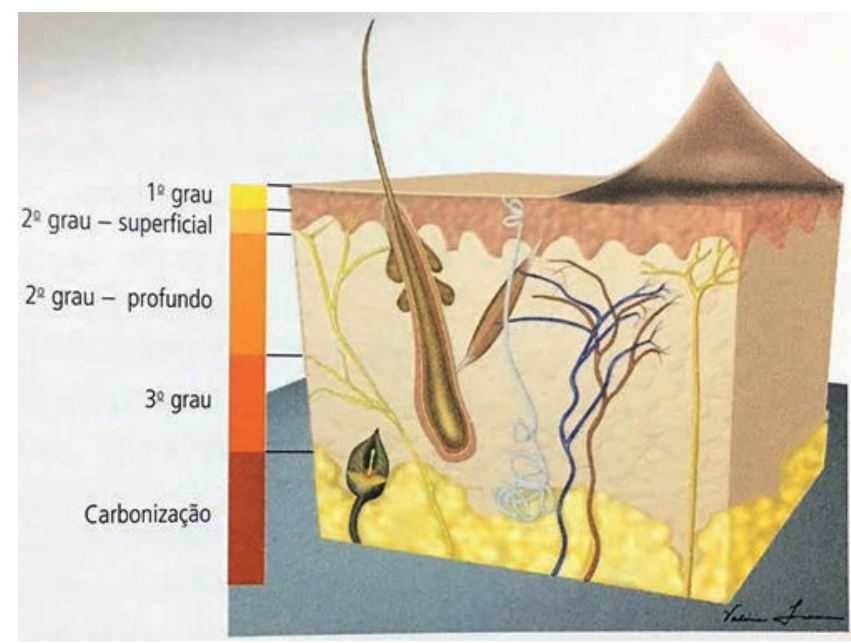

Figura 1 - Representação dos graus de Queimadura e relação com a profundidade

Fonte: GUIRRO e GUIRRO (2007, p. 493)

\section{Microagulhamento}

\section{História}

Microagulhamento, uma forma de terapia da indução do colágeno, foi introduzido em 1997 como uma modalidade nova do tratamento usando um dispositivo chamado Dermaroller. Ele foi usado com indicação para tratamento de cicatrizes e rugas. Importantes marcos no desenvolvimento da técnica: 1995 - Orentreich e Orentreich descreveram subcisão para o tratamento de cicatrizes e rugas. 1997 - Camirand e Doucet descreveram a técnica utilizando pistolas de tatuagem, sem pigmento, após procedimento cirúrgico na face, observando que houve uma "quebra" e realinhamento do colágeno, neovascularização, normalização na coloração da pele, além de tornar a cicatriz mais suave, flexível e menos evidente. 2006 Fernandes desenvolveu a terapia percutânea da indução do colágeno (PCI) com Dermaroller para iniciar a cascata inflamatória traumática do crescimento natural por rolamento de agulhas verticalmente, horizontalmente e diagonalmente, com pressão sobre a área tratada (NAIR, 2014)

\section{Caneta Eletrônica}

Caneta eletrônica ou Derma Erase Pen ${ }^{\circledR}$, como é também comercializada, é uma caneta elétrica contendo inúmeras microagulhas para serem utilizadas apenas durante a sessão. É descrita como um dispositivo de microagulhamento, com um anel do ajuste que permite a alteração das alturas das microagulhas. O microagulhamento é uma terapia considerada segura por provocar danos mínimos à pele, resultando em muito menos lesão epidérmica do que o observado com outros métodos. Há também um menor risco de hiperpigmentação e cicatrizes com microagulhamento, sendo uma escolha apropriada de tratamento para pessoas com pele fina, sensível e de diferentes fototipos (AMER, 2018).

A caneta eletrônica para microagulhamento, apesar de vendida há algum tempo, só obteve 
registo na ANVISA para utilização no Brasil em 2019. Ela permite a regulagem do tamanho das agulhas entre $0,25 \mathrm{~mm}$ e 2,0 $\mathrm{mm}$ (Figura 2), de acordo com a profundidade da afecção.

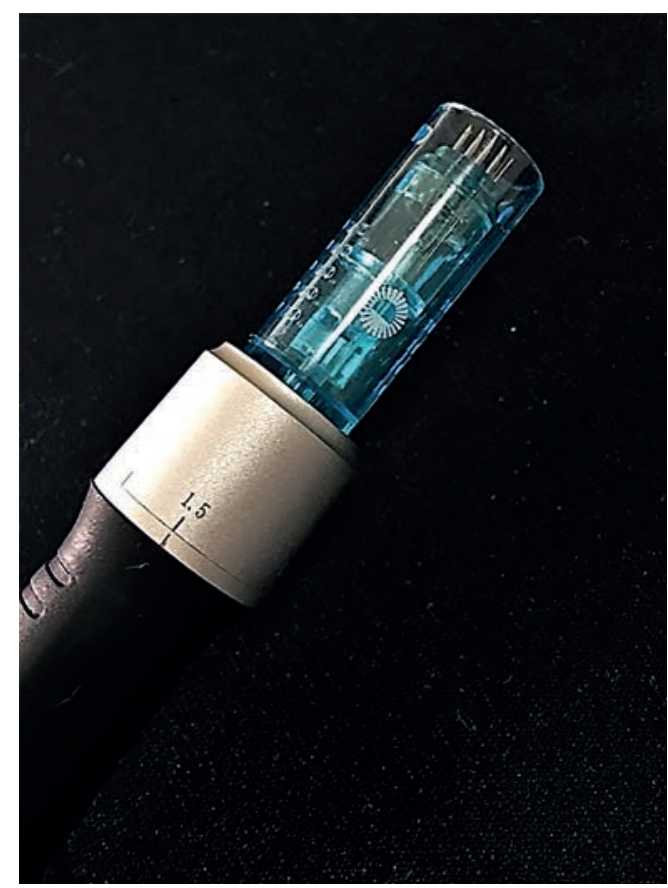

Figura 2 - Sistema de ajuste do tamanho das agulhas em caneta eletrônica

Fonte: Arquivo pessoal da autora

A caneta eletrônica promove um movimento na vertical de forma automatizada. O sistema elétrico faz com que as agulhas subam e descam automaticamente, de acordo com o ajuste da profundidade realizado anteriormente na caneta, sem a necessidade de pressioná-la sobre a pele (BORGES, 2016). Segundo o Manual do Usuário (DERMA ERASE PEN, 2017), recomenda-se manter a caneta a $90^{\circ}$ em relação à pele para que as agulhas perfurem de forma linear e homogênea.

\section{Efeitos Fisiológicos}

A técnica age, basicamente, de duas maneiras: estimulando a produção natural do colágeno (PNC) ou indução percutânea de colágeno através da resposta do processo inflamatório, 0 que facilita o Sistema de Acesso Transdérmico de Ingrediente (SATI), conhecido como "Drug Delivery" (aumento na permeação de ativos) (NEGRÃO, 2017).
As microagulhas penetram na epiderme, causando microlesões controladas e localizadas. Apesar das microlesões, a barreia de proteção da pele permanece íntegra, evitando problemas como hiperpigmentação ou despigmentação, por exemplo. A partir da aplicação da IPC, inicia-se imediatamente o processo de reparação, objetivando a restauração do tecido lesado, que consiste numa sequência de eventos moleculares, divididos em três fases que se sobrepõem de forma temporal e contínua (FERNANDES, 2008).

A Indução Percutânea de Colágeno (IPC) começa com a perda da integridade da barreira cutânea, tendo por resultado a liberação das citocinas, tais como o interleucina- $1 \alpha$ (predominante), o interleucina-8, Interleucina-6, Fator de Necrose Tumoral- $\alpha$ (TNF- $\alpha$ ) e Fator Estimulante de Colônias de granulócitos-macrófagos (GM-CSF), levando à vasodilatação dérmica e migração de queratinócitos, um processo que restaura o dano epidérmico. Três estágios de reparação após trauma com agulhas podem ser claramente delineados: na primeira etapa, fase inflamatória, há liberação de plaquetas e neutrófilos, que são responsáveis por liberar fatores de crescimento que atuam sobre queratinócitos e fibroblastos como fatores de crescimento e transformação $\alpha$ e $\beta$ (TGF $\alpha$ e TGF- $\beta$ ), de fator de crescimento derivado de plaquetas (PDGF), da proteína III (ativador do tecido conjuntivo) e do fator de crescimento do tecido conjuntivo (LIMA, 2013).

Na segunda fase, proliferativa, os neutrófilos são substituídos por monócitos, e a angiogênese, a epitelização e a proliferação de fibroblastos acontecem, seguidas da produção de colágeno tipo III, elastina, glicosaminoglicanos e proteoglicanos (LIMA, 2013).

Concomitantemente, o fator de crescimento dos fibroblastos, TGF $\alpha$ e TGF- $\beta$ são secretados por monócitos. Aproximadamente cinco dias após a lesão, a matriz de fibronectina é completamente formada, permitindo a deposição de colágeno diretamente abaixo da camada basal da epiderme (LIMA, 2013). 
Na terceira fase, de remodelamento ou maturação, o colágeno tipo III, que prevalece na fase inicial do processo de cicatrização, é lentamente substituído pelo colágeno tipo I (que dura mais tempo e persiste por um período variando de cinco a sete anos). Para que essa sequência inflamatória de eventos ocorra, o trauma deve ser causado por uma agulha que atinja de $1 \mathrm{~mm}$ a $3 \mathrm{~mm}$, e a epiderme deve ser preservada (apenas perfurada e não removida). Centenas de microlesões são criadas, resultando em colunas de sangue coletadas na derme, acompanhadas de edema da área tratada e hemostasia praticamente imediata. A intensidade dessas reações é proporcional ao comprimento da agulha utilizada no procedimento. Por exemplo, $1 \mathrm{~mm}$ profundidade implica um hematoma quase microscópico, enquanto o resultante de uma profundidade de $3 \mathrm{~mm}$ pode ser visto a olho nu e pode persistir por horas. No entanto, é necessário compreender que a agulha não penetra completamente durante o processo de laminação (LIMA, 2013).

Para Setterfield (2013), assim como para outros autores, a chave da técnica está no processo de regeneração da pele, assim como outros autores, mas enfatiza e explica o mecanismo de comunicação celular baseado num comparativismo entre a regeneração do embrião, que se isenta de cicatriz, e a regeneração adulta, na qual não há falta de cicatriz.

\section{Registro e Análise dos Dados}

Antes de cada sessão, foram registradas imagens da cicatriz e efetuada a caracterização dela utilizando-se a Escala de Cicatrização de Vancouver, utilizada para avaliar a pigmentação, vascularização, flexibilidade e altura da cicatriz. Os dados obtidos a partir dessa escala foram analisados em cada um dos três momentos (antes de cada sessão) e dois meses após a última sessão.

A escala de Vancouver tem pontos que variam de $\mathrm{O}$ a 13. Quanto menor a somatória da pontuação final, melhor apresenta-se o estado geral da cicatriz de acordo com as carac- terísticas avaliadas. Seguem itens e pontuações avaliadas: (a) Pigmentação: $0=$ Normal - Coloração similar à cor do resto do corpo; 1 = Hipopigmentação; 2 = Hiperpigmentação. (b) Vascularização: $0=$ Normal - Coloração similar ao resto do corpo; 1 = Rosada; 2 = Avermelhada; 3 = Púrpura. (c) Flexibilidade: 0 = Normal; 1 = Maleável-flexível à mínima resistência; 2 = Deformação - cede sob pressão; 3 = Firme-inflexível, não move facilmente, resistente a pressão manual; 4 = Bandas - tecido na forma de cordas com coloração esbranquiçada em sua extensão; 5 = Contratura - encurtamento permanente da cicatriz, produzindo deformidade ou distorção. (d) Altura: $0=$ Normal; $1=<$ $2 \mathrm{~mm} ; 2=<5 \mathrm{~mm} ; 3=>5 \mathrm{~mm}$.

Foi realizada fotodocumentação em diferentes momentos do tratamento, como descrito anteriormente, visando registrar qualitativamente os efeitos da IPC sobre as sequelas de queimadura tratadas.

Foram analisados também os dados obtidos a partir da Escala Visual Numérica, utilizada para avaliar a sensibilidade/dor em relação ao procedimento, também referida anteriormente neste protocolo.

Foi realizada avaliação cega por oito avaliadores, docentes de duas instituições de ensino superior em São Paulo, para avaliar a melhora da pigmentação da pele.

\section{Estudo de Caso}

Voluntária, 54 anos, queimadura por óleo quente, há dois anos, região do membro superior esquerdo, $2^{\circ}$ grau profundo. Os dados analisados estão demonstrados a seguir:

A escala de Vancouver apresentou melhora de $43 \%$ no resultado (7 para 4), sendo essa melhora relativa à flexibilidade (-1), vascularização (-1) e altura (-1). A Escala Visual Numérica apontou aumento de 1 ponto (3 para 4), demonstrando aumento da sensibilidade local. A avaliação cega apontou melhora de $36 \%$ em relação à pigmentação (Figura 3), conforme imagem antes e depois apresentada a seguir: 


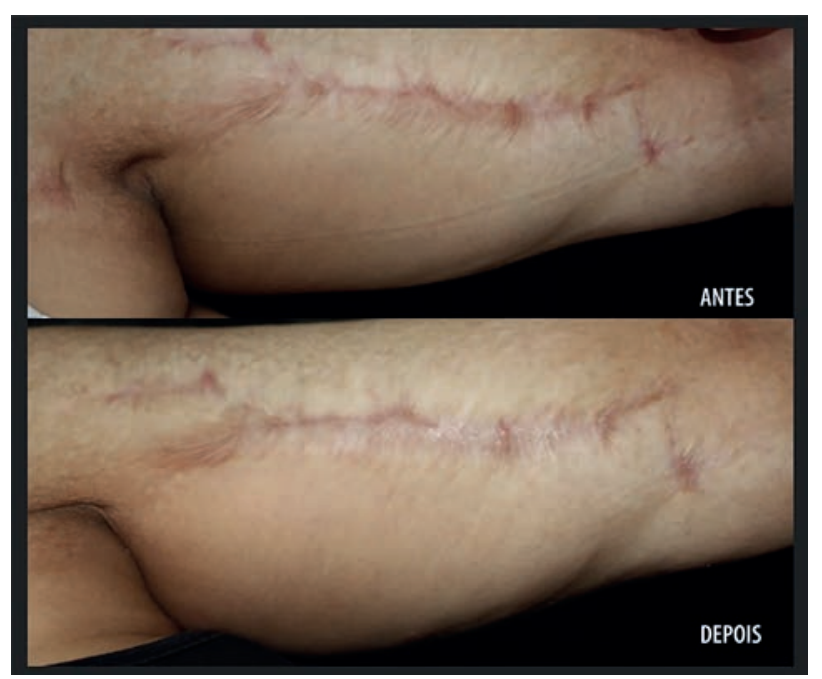

Figura 3 - Participante 5 - MSE

Fonte: Arquivo pessoal da autora

Podemos observar (Gráfico 1) que a melhora da região foi principalmente à escala de Vancouver, relacionada à flexibilidade, vascularização e altura da cicatriz.

\section{Gráfico 1 - Resultados Avaliação Cega e Es- cala de Vancouver}

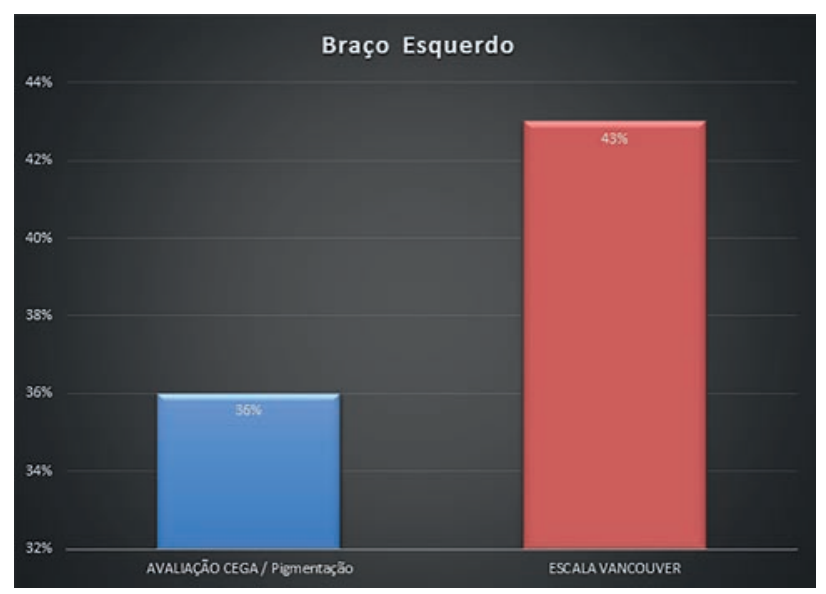

Fonte: Elaborado pela autora

\section{Considerações Finais}

A partir dos resultados obtidos, conclui-se que a Indução Percutânea de Colágeno por técnica de microagulhamento, utilizando-se caneta eletrônica, é um tratamento que melhora significativamente a aparência geral das cicatrizes causadas por queimaduras, permitindo ganhos mensuráveis quanto à vascularização, flexibilidade, sensibilidade, pigmentação e altura da cicatriz.
Apesar dos diferentes graus de melhora para cada quesito analisado, houve melhora significativa das cicatrizes e, consequentemente, da autoestima e qualidade de vida da participante, constatado por depoimento voluntário captado.

Sugere-se a realização de novos estudos com grupos de voluntários maiores e em diferentes regiões que venham agregar ainda mais para a comunidade acadêmico-científica.

\section{Referências}

AMER, M. et al. Dermapen in the treatment of wrinkles in cigarette smokers and skin aging effectively. J Cosmet Dermatol, v. 17, p. 1.2001.204, 2018.

AZULAY, R. D. Dermatologia. 7. ed. Rio de Janeiro: Guanabara Koogan, 2017.

BOLGIANI, A. N.; SERRA, M. C. V. F. Updating in the local treatment of the burns. Rev. Bras. de Queimaduras, v. 9, n. 2, p. 38-44, 2010.

DERMA ERASE PEN®. Manual do Usuário. Versão: V2.0.2. 30 ago. 2017.

FERNANDES, D.; SIGNORINI, M. Combating photoaging whit percutaneous collagen induction. Clin. Dermatol., v. 26, n. 2, p. 192199, 2008.

FERREIRA, L. M. (org.). Cirurgia Plástica. Barueri, SP: Manole, 2007.

GUIRRO, E.; GUIRRO R. Fisioterapia Dermato funcional: fundamentos, recursos, patologias. 3. ed. Manole: São Paulo, 2007.

LIMA, E. V. A; LIMA, M. A.; TAKANO, D. Microagulhamento: estudo experimental e classificação da injuria provocada. Surg. Cosmet. Dermatol., v. 5, n. 2, p. 110-114, 2013.

METSAVAHT, L. Queimaduras e suas cicatrizes. Surg. Cosmet. Dermatol., v. 9, n. 4, p. 281-284, 2017.

NAIR, P. A.; ARORA, T. H. Microneedling using dermaroller: a means of collagen induction therapy. Gujarat Med J., v. 69, n. 1, p. 24-27, 2014.

NEGRÃO, M. M. C. Microagulhamento: bases fisiológicas e práticas. 2. ed. São Paulo: CR8 Editora, 2017. 
PETRI, V. Dermatologia Prática. Rio de Janeiro: Guanabara Koogan, 2009.

RIBEIRO, D. Queimaduras. In: PEREIRA, M. F. L. (org.). Recursos técnicos em estética. São Caetano do Sul, SP: Ed. Difusão, 2013. v. 2.

SILVA, V. C. C. Tratamento estético no queimado. In: BORGES, F. S.; SCORZA, S. A. (org.). Terapêutica em estética: conceitos e técnicas. São Paulo: Ed. Phorte, 2016.
STEFANELLI, R. Queimaduras. In: FERIANI, G. et al. (ed.). GRAU - Pré-Hospitalar. 2. ed. São Paulo: Manole, 2015.

VALE, E. C. S. Primeiro atendimento em queimaduras: a abordagem do dermatologista. An. Bras. Dermatol., v. 80, n. 1, p. 9-19, 2005.

VANA, L. P. M. Sequelas de queimaduras: nova classificação. Rev. Bras. Queimaduras, v. 12, n. 3, p. 192-2, 2013. 